\title{
A research on the development of the resolution improvement methods in electron microscopy
}

\author{
Nana Gao ${ }^{1, ~ a ~}$, Lijing $\mathrm{Li}^{1, \mathrm{~b}}$, Zhihui Tie ${ }^{1, \mathrm{c}}$ and Yongqin Wan ${ }^{1, \mathrm{~d}}$ \\ ${ }^{1}$ School of instrumentation science and opto-electronics engineering, Beihang University, Beijing \\ 100191, China. \\ agaonanay@163.com, ${ }^{b}$ lilj@eyou.com, ‘Xinglai11@outlook.com, dsusan2012_ok@126.com
}

Keywords: Electron microscopy, resolution, spherical aberration, chromatic aberration.

\begin{abstract}
Influencing factors of electron microscopy spatial resolution and information resolution were reviewed in this paper. The methods to improve the resolution of electron microscopy were discussed from the aspects of choice of electron gun, spherical aberration correction, chromatic aberration correction and high-performance detection device etc. Besides, examples were given to illustrate the effects of the method adopted. Meanwhile, the key method to obtain further resolution of electron microscopy is to work out a corrector compensating the chromatic aberration of the objective lens.
\end{abstract}

\section{Introduction}

Electron microscopy broke the diffraction limit (200nm) of the optical microscope using electron beam as the light source and the human vision was expanded to atomic level. High resolution electron microscopy is the guarantee for the information of atom structure details, chemical composition and the structure of the non-periodic atomic structure. In 1931, German physicists, Knoll and Ruska developed a prototype of transmission electron microscopy with two magnetic lenses achieving the resolution of $50 \mathrm{~nm}$. After 80 years' development, the resolution of the electron microscopy has been developed into sub- $\AA$ level. The newly released product JEM-ARM300F by Japanese company JEOL reached $0.063 \mathrm{~nm}$ resolution making a breakthrough progress in the field of electron microscopy. However, in order to obtain the interatomic bonding information, efforts must be continuously made to eliminate all kinds of aberrations in electron microscopy [1, 2].

\section{Improvement of the resolution in electron microscopy}

According to different imaging modes, electron microscopy can be divided into scanning electron microscopy (SEM) and transmission electron microscopy (TEM). Scanning electron microscopy continues to use the definition of the spatial resolution in ordinary optics microscopy. Otherwise, transmission electron microscopy resolution cannot be fully reflected by spatial resolution [3]. Therefore, information resolution is introduced into TEM defining a minimum visible information in case of symmetric lighting [4].

The spatial resolution is defined under the condition of Scherzer theory, thin object thickness, weak phase object and good electron monochromaticity:

$$
\delta=0.65 C_{s}^{\frac{1}{4}} \lambda^{\frac{3}{4}}
$$

In equation (1), $C_{s}$ is the aberration coefficient of objective lens, $\lambda$ is electron wavelength. The relationship between electron wavelength and electron accelerating voltage is calculated as below considering the relative effects of the speed of light:

$$
\lambda=\frac{1.225}{\sqrt{V}}\left(1+0.978 \times 10^{-6} V\right)^{\frac{1}{2}}
$$

Combined equation (1) and (2): the electron microscopy resolution is a function of spherical coefficient and electron wavelength. On one hand, small spherical aberration coefficient and high 
accelerating voltage can obtain high image resolution, on the other hand, stable accelerating voltage and small electron beam wavelength range can guarantee better stability of the resolution of electron microscopy.

The information resolution is reciprocal of the highest spatial frequency of the electron contrast transfer function from object plane to image plane. It is the minimum information achieved by image reconstruction [3] which is controlled by the electron wavelength and the focusing diffusion caused by chromatic aberration focusing. The focusing difusion can be expressed as below:

$$
\Delta f=C_{c} \sqrt{\left(\left(\frac{\Delta V}{V}\right)^{2}+\left(2 \frac{\Delta I}{I}\right)^{2}+\left(\frac{\Delta E}{E}\right)^{2}\right)}
$$

In equation (3), $C_{c}$ is the chromatic aberration coefficient of objective lens, $\Delta V / V$ is the relative fluctuation of accelerating voltage for electron gun, $\Delta I / I$ is the relative fluctuations of the objective current and $\Delta E / E$ is the relative diffusion of the electron beam energy.

In summary, electron microscopy resolution is mainly affected by the performance of electron source, the stability of accelerating voltage and objective lens' current, the spherical aberration and chromatic aberration in objective lens as well as the energy dispersion of electron beam. Besides improving the configuration of the hardware parts in electron microscopy, we should also use high-performance detection technology to obtain high resolution.

Selection of electron gun. The electron gun with long life, high brightness, high energy distribution and good mechanical stability is the guarantee of high resolution in electron microscopy.

Hot tungsten filament cathode electron gun is the earliest invention used in electron microscopy, but it wasn't used widespread due to its low axis brightness, high energy dispersion and low cathode current density. In 1969, Broers proposed the first practical $\mathrm{LaB}_{6}$ electron gun, cathode emission current density was increased to $65 \mathrm{~A} / \mathrm{cm}^{2}$, shaft brightness was increased to $10^{6} \mathrm{~A} / \mathrm{cm}^{2} \cdot \mathrm{Sr}$ and energy dispersion was decreased to $1 \mathrm{eV}$ [5]. During this period, the cold field emission electron gun proposed by Crewe in 1968 also had a great development, axial brightness reached more than $10^{9} \mathrm{~A} / \mathrm{cm}^{2} \cdot \mathrm{Sr}$, cathode current density reached $10^{4} \mathrm{~A} / \mathrm{cm}^{2}$, energy dispersion was reduced to $0.3 \mathrm{eV}$ with very small $-0.1 \mu \mathrm{m}$ radius of tip curvature. Then it was put into application in 1980 [6]. However, the problems of small emission current and instability in cold field emission gun prompted Swanson [7] et al to study the extended schottky emission electron gun in the 1970s. Although the radius of tip curvature is larger than that of cold field emission electron gun, it avoided the problem of small emission current and the instability in cold field emission electron gun.

Since mid-nineties of the last century, cold field emission electron gun and extended schottky electron gun was extensively used [8]. The cold field emission electron gun was used in the newly released product JEM-ARM300F, JEM-ARM200F and other high-resolution electron microscopy manufactured by company JEOL. Meanwhile, JEM-ARM200F was also equipped with schottky emission electron gun. They both achieved sub- $\AA$ resolution. The latest research results from Japanese scholars Fujita and Shimoyama showed that apparent brightness of field emission gun decreased instead of increasing as a result of the increase of the apparent diameter of intersection when electron beam $\left(\mathrm{I}_{\mathrm{b}}\right)$ exceeded the threshold current. In that case, the performance of field emission electron gun is not as good as hot emission electron gun [8], which provides an important reference in the application of SEM electron gun in the future.

The spherical aberration correction. The boundary effect of rotationally symmetric electromagnetic lens makes the focal points of the large aperture angle electrons and the small aperture angle electrons that from one objective point different, which contributes to a dispersed pellet near the ideal image point. The dispersion pellet limits the resolution in electron microscopy [9], causing the image sharpness reduced and the quality of image declined. Therefore, the diameter of the round spot caused by spherical aberration must be reduced in order to improve the resolution of electron microscopy. 
In the early period of electron microscopy, reducing lens pole spacing once as a means of reducing the spherical aberration was widely used. However, the spherical aberration of the objective lens was close to the limit values because of the sample space constraints [10]. Spherical aberration corrector composed of multipolar electromagnetic lens and auxiliary lens has become a useful means to reduce spherical aberration coefficient through producing negative spherical aberration compensation. Early in 1936, Scherzer [11] pointed out: in these three conditions (1) electron optics system is rotationally symmetric; (2) lens field is static; (3) there is no space charge in the light path, the spherical aberration and chromatic aberration of the objective lens is difficult to eliminate. One of the three conditions must be broken to design spherical aberration corrector. Meanwhile, introducing a multi-pole field unit in the light path to break the rotationally symmetric structure in electromagnetic lens is the primary way [12].

In order to correct the spherical aberration coefficient of objective lens, Zach and other scientists have developed a variety of spherical aberration corrector's structure. In 1995, Zach and Haider successfully used quadrupole and octupole electromagnetic field to compensate the spherical and chromatic aberration at the same time in low voltage scanning electron microscopy (LVSEM). As a result, the resolution of LVSEM in $1 \mathrm{kV}$ accelerating voltage was improved from $5 \mathrm{~nm}$ to $1.8 \mathrm{~nm}$ [13]. The large off-axis aberration made it not suitable for the spherical aberration correction of TEM which requires large field of view. In order to obtain a sufficiently large field of view in the lens, Rose presented a pure magnetic sextupole spherical aberration correction system which was successfully designed in 1995, its structure as shown in figure 1 [14] includes three parts: (1) a first transfer lens group composed of two circular lens; (2) two sextupole field units; (3) a second transfer lens group between the two sextupole field unit.

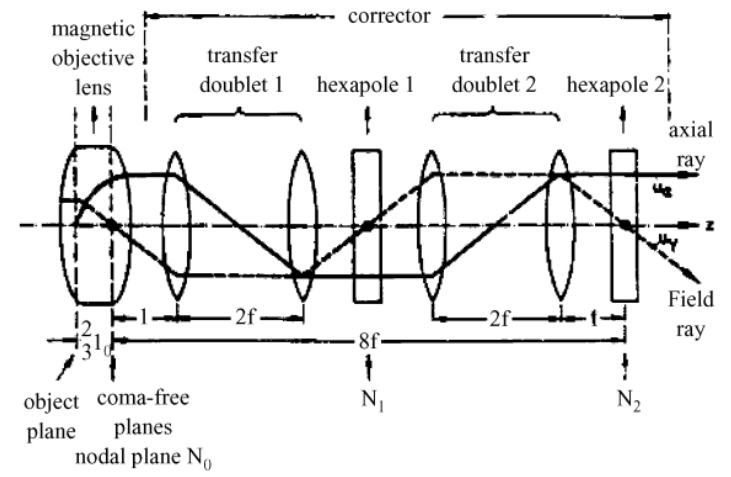

Fig.1 Sextupole spherical aberration correction system by Rose

The third order spherical aberration coefficient of the CM200 FEG ST type TEM was decreased to $50 \mu \mathrm{m}$ with the sixtupole spherical aberration corrector, meanwhile the resolution was improved from $0.24 \mathrm{~nm}$ to $0.13 \mathrm{~nm}$. Even if the pure magnetic sextupole field can only correct the lens' spherical aberration, its $10 \mathrm{ppm}$ stability was easy to realize compared with the $0.5 \mathrm{ppm}$ stability requirement of the electromagnetic quadrupole field in $0.12 \mathrm{~nm}$ [13]. At present, Japanese company JEOL used the ETA-twelve pole spherical aberration corrector in its new released product JEM-ARM300F achieving the ultrahigh resolution of $0.063 \mathrm{~nm}$ in $300 \mathrm{kV}$ accelerating voltage.

Chromatic aberration correction. The focusing properties of the electron optics system in electron microscopy for different speed electrons are different. Therefore the changes of electron wavelength caused by the fluctuations in accelerating voltage and the changes of the focusing properties caused by the fluctuations in magnetic lens current will both make the image blurred, resulting in chromatic aberration [9].

The chromatic aberration of electron microscopy can only be corrected by electrostatic and magnetic units. However, this complex field hasn't been successfully produced because of the high stability requirement until now. The improvement of stability of the accelerating voltage and the lens current are both helpful to the suppression of the electron beam energy dispersion. Both of them have reached the level of $10^{-6} / \mathrm{min}$ at present. In addition, monochromator is also an effectively means to reduce the energy dispersion of electron beam. Monochromator generally includes a reducer, an 
energy scattering component, an accelerator and energy selecting slits. In monochromator, different energy electrons are separated first and then energy selection unit selects a certain spectral width of the electron beam reducing the energy spread of electron beam. Monochromator can be classified into four types according to different working principles and structure: retarding Wien Filter [15], Fringe-Field monochromator [16], Omega-type filter [17] and Mandoline energy filter [18]. Omega-type filter can be divided into magnetic field and electrostatic field type monochromator [3]. It is difficult to realize high spatial resolution for Wien type monochromator due to its unsatisfied spot brightness and contrast of electron beam causing by the distinct Coulomb force between the electrons [19, 20]. Fringe-Field monochromator designed by Mook and Kruit in order to reduce the Coulomb force $[16,21]$ can reduce the energy dispersion to $0.05 \sim 0.1 \mathrm{eV}$ by using $200 \mathrm{~nm}$ energy selecting slits [3]. Omega-type monochromator proposed by Rose and Kahl avoided the lateral Coulomb force, eliminated the energy dispersion and obtained high brightness electron beam with its good symmetrical structure [3]. The Mandoline energy filter proposed by Uhlemann and Rose [22] effectively eliminated the aberration of the filter. It achieved $0.2 \mathrm{eV}$ energy spectral width by Fransen et al in 1999 with carbon nanotubes in the ends of the tungsten filament [23]. Omega-type and Mandoline-type monochromator are commonly used in recent years.

Freitag et al. configured TEM with Mandoline monochromator making the energy spectrum width reduced from $0.6 \mathrm{ev}$ to $0.15 \mathrm{ev}$. Meanwhile the information resolution of TECNAI F20 S2Twin was improved from $0.11 \mathrm{~nm}$ to $0.09 \mathrm{~nm}$ with a monochromator through calculation [3]. Figure 2 shows the interference fringes of the non-crystalline tungsten thin film when Mandoline monochromator is off and on, in which the small, dark circle shows the spatial frequency corresponding to $0.14 \mathrm{~nm}$, the big, light circle shows the spatial frequency corresponding to $0.1 \mathrm{~nm}$, making information resolution up to $0.1 \mathrm{~nm}$ level $[24,25]$. Although the configuration of monochromator improved the energy resolution of the electron microscopy to $0.1 \mathrm{eV} \sim 0.2 \mathrm{eV}$, the electron beam with monochromator was less than one tenth of that without monochromator due to the energy filtering effect [26] which significantly reduced the brightness of electron beam [9]. Considering this, energy dispersion and electron beam should both be considered in the selection of the optimum width of the monochromator energy filtration slits.
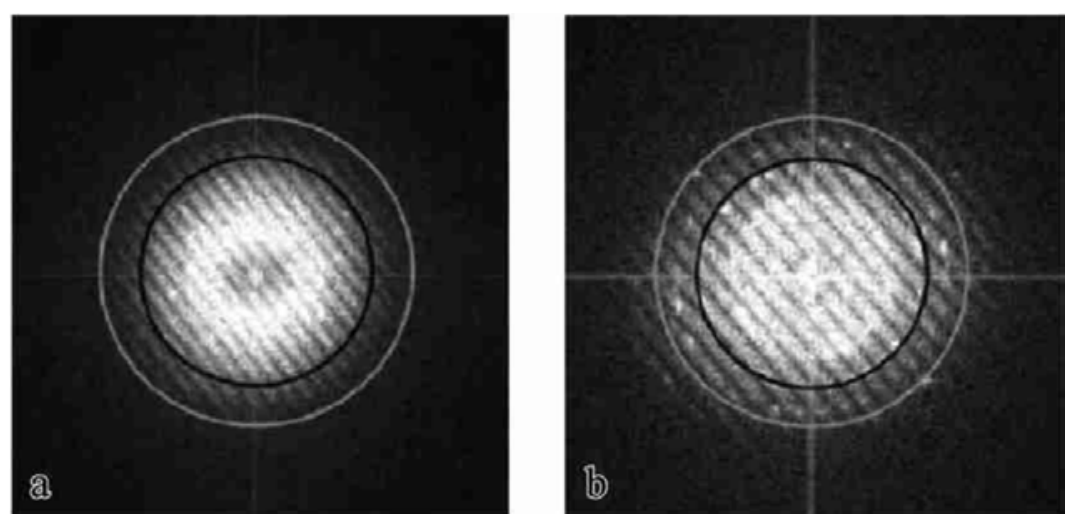

Fig. 2 Two Young's fringe images of an amorphous tungsten film by Freitag et al
a. Monochromator off $(\Delta E=0.6 \mathrm{eV})$ and ${ }^{C_{s}}$ corrector on $\left({ }^{C_{s}}=-3 \mu \mathrm{m}\right)$
b. Monochromator on $(\Delta E=0.15 \mathrm{eV})$ and $C_{s}$ corrector on $\left(C_{s}=-3 \mu \mathrm{m}\right)$

High performance detection means. In order to improve the resolution of electron microscopy, on one hand, it needs to improve the design of electron microscopy. On the other hand, it also needs to rely on better detection means to ensure high quality images. CCD imaging technology provides a technical support for obtaining high resolution images with high sensitivity and high signal to noise ratio. In recent years, the operation of the electron microscopy is controlled by computer and the image acquisition work has also started to use CCD camera to replace the traditional photographic film. In 2002, the UltraScan1000 SSCCD produced by Gatan met the target of real-time observation whose pixel size was $14 \mu \mathrm{m}$, the number of pixels was $2048 \times 2048$, size of field is $28.7 \mathrm{~mm} \times 28.7$ $\mathrm{mm}$, speed of data reading and writing are $4 \mathrm{MHz}$ [9]. At the beginning of 2006, a new CCD 
832SC1000W with 11 million pixels launched by Gatan which can record dynamic image is the first and the only one CCD camera to capture electron diffraction directly [26]. The improvement of the aberration correctors combined with high performance digital detection method-CCD make electron microscopy can not only obtain high quality image, acquire and analysis the data of diffraction as well as the $3 \mathrm{D}$ reconstruction function, but also can record the process of the evolution of the structure on the atomic scale.

\section{Summary}

The theory resolution of electron microscopy is affected by spherical aberration, chromatic aberration and many other factors. The spherical aberration correctors and the development of the energy dispersed suppression technology have made the resolution of electron microscopy up to sub-Å level. JEM-ARM300F, a new released STEM by Japanese company JEOL, has realized the ultrahigh resolution of $0.063 \mathrm{~nm}$, which marks the top level of the present resolution. In 2004, TEAM project group had started the redesign work of the conventional sixtupole spherical aberration corrector, aiming to compensate chromatic and spherical aberration in one corrector at the same time in order to achieve $0.05 \mathrm{~nm}$ resolution in STEM and TEM [12]. Although such an attempt is full of challenges, it can't be despised. I believe that this corrector will be successfully put into application on STEM and TEM with the development of technology in the near future.

\section{References}

[1] Yao Junen, Present situation and prospect of electron microscopy, J. Electron microscopy. 17, 1998 (6).

[2] H Rose, Optics of high-performance electron microscopes, J. Science and technology of advanced materials. 2008.

[3] Zhou Yusheng, Wang Rongming, Shang Guangyi, Yao Junen, The development and application of TEM monochromator, J. Journal of microscopy. 2009, 28 (1).

[4] Tang Dong, B. Freitag, Improvement of TEM resolution, J. Electron microscopy. 2004, 23 (4).

[5] A N Broers, Electron and ion probes. In Proceedings of the 5th International Conference on Electron and Ion Beam Science and Technology. 1972 , P3-25.

[6] A V Crewe, et al. Rev Sci Instrum, Convergent beam electron diffraction-a novel technique for materials characterisation at sub-microscopic levels, J. 1968, 39: 576.

[7] L A Swanson, N A Martin, J of Applied Physics, 1975, 46(5): 2029.

[8] Chen Wenxiong, Xu Jun, Zhang Huizhen, Chen Li, New progress in the study of electron gun theory, J. Electron microscopy. 2006, 25 (6).

[9] Zhu Zufu, Shen Jinde,, Xu Zhiyi, Lu Guohui, Zhuang Dianliu, Electron micorocope, M. China machine press.

[10] Li Douxing, Progress of transmission electron microscopy I Development of transmission electron microscope and related equipments, J. Journal of microscopy. 2004, 23 (3).

[11] Scherzer O, Aberration-corrected HREM/STEM for semiconductor research, J. Z Phys. 1936, 101: 593.

[12] Haider M, Hartel P, Müller H, Uhlemannand S. Zach J, Current and future aberration correctors for the improvement of resolution in electron microscopy, J. Philosophical transactions of the royal society. 2009, 367:3665-3682. 
[13] Chen Wenxiong, Xu Jun, Chen Li, Zhang Huizhen, Correction of spherical aberration of a medium voltage TEM, J. Journal of electron microscopy. 2007, 26 (1).

[14] Haider M, Braunshausen G, Schwan E. Optik. 1995, 99(4)167.

[15] Mook H W, Kruit P, Electrostatic in-line monochromator for Schottky Field emission gun, J. Inst.Phys. Conf.Ser. 1997, 153, 2: 81-84.

[16] Mook H W, Kruit P, Optics and design of the fringe field monochromator for a Schottky field emission gun, J. Nuclear Instruments and Methods in Physics Research A. 1999, 427:109-120.

[17] Rose H, Prospects for realizing a sub-A sub-eV resolution EFTEM, J. Ultramicroscopy. 1999, 78:13-25.

[18] Essers Erik, Dieter Krahl, Bernd Huber, et al, Performance of corrected imaging energy filters, J.

[19] Tiemeijer P C, Measurement of Coulomb interactions in an electron beam monochromator, J. Ultramicroscopy. 1999,78: 53-62.

[20] Den Dekker A J, Van Aert S, Van Dyck D, et al, Does a monochromator improve the precision, J. Ultramicroscopy. 2001, 89:275-290.

[21] Mook H W, Kruit P, The introduction of monochromators in electron microscope guns, J. Journal of electron microscopy. 2000, 19:113-118.

[22] Uhlemann S, Rose H, The Mandoline filter-a new high-performance imaging filter for sub-eV EFTEM, J. Optik.1994, 96, 44:163-178.

[23] FransenM J, Van Rooy T L, Kruit P, Field emission energy distribution from individual multi-walled carbon nanotubes, J. Appl Surf Sci. 1999 , 146:312-327.

[24] Fejes P L, Approximations for the calculation of high-resolution electron microscope images of thin films, J. ActaCryst. 1977, A33, 1: 109-113.

[25] Freitag B, Kujawa S , Mul P M , et al, Breaking the spherical and chromatic aberration barrier in transmission electron microscopy, J. Ultramicroscopy. 2005, 102: 209-214.

[26] Zhang Detian, Liu Ansheng, Zhu Yanyong et al, Development trend and application characteristics of electron microscopy, J. 